http://jmscr.igmpublication.org/home/ ISSN (e)-2347-176x ISSN (p) 2455-0450

crossref DOI: https://dx.doi.org/10.18535/jmscr/v7i12.63

Journal Of Medical Science And Clinical Research

\title{
Post sensitization assessment of knowledge attitude and practice regarding clinical sample collection, storage and transportation among Health care workers at a tertiary care Hospital in central Madhya Pradesh
}

\author{
Authors \\ Dr Nidhi Sharma ${ }^{1}$, Mrs. Hirdesh Kumari Gupta ${ }^{2}$, Dr Kiran Tripathi ${ }^{3}$ \\ ${ }^{1}$ Asst Prof. Department of microbiology, GMC Datia \\ ${ }^{2}$ Tutor Department of Microbiology, Datia \\ ${ }^{3}$ Prof \& Head Department of Microbiology GMC Datia
}

\section{Introduction}

Specimen collection plays an important role in getting timely and accurate results of investigation required for diagnosis. If there is any error in delivery of the sample from patients to laboratory, which might be due to improper collection, untimely collection, poor transportation, it will lead to error in diagnosis ${ }^{(1)}$ and most of these mistakes are human and mostly at pre-analytical level which are avoidable. Among them HCW play an important role who provide first hand bedside care and their action have a direct role in positive patient outcome as it affects sample quality which is processed in laboratory

It is therefore important to develop systems and routine to enhance knowledge, attitude and practice among health care workers regarding proper sample collection, storage and transportation $^{(2)}$

\section{Materials and Method}

A cross sectional study was conducted among the health care workers of a tertiary care hospital in Central Madhya Pradesh to assess their knowledge regarding safe and proper sample collection and transportation. Total 15 health care workers participated in this study. A pre session questionnaire was designed to enquire their knowledge regarding collection of sample, its timing, storage, transportation and report turnover time. A special mention was given to sample collection in case of tuberculosis and also about disinfection.

Consent was taken from participants to taking part in this questionnaire study. The mean time for completing that questionnaire was 10 minutes. Confidentiality was assured to participants and strictly maintained during this study.

A session of $45 \mathrm{~min}$ to enhance their knowledge in above mentioned all aspects was taken.

A post session questionnaire with same questions was distributed and their knowledge after session was checked in $10 \mathrm{~min}$.

\section{Result}

Pre - session

Gross deficiency was found among the healthcare workers regarding KAP in the area of proper sample collection techniques, handling of sample, transportation and storage of sample, dealing with blood spillage and in general disinfection. 
About $27 \%$ health care workers have the knowledge regarding proper ways to collect sample. Only 7\% HCW know when to collect sample. $27 \% \mathrm{HCW}$ have the idea of how to store sample and about $13 \%$ regarding their transportation. Only $27 \% \mathrm{HCW}$ know when to expect report of test or the time taken to process the samples.

About $47 \%$ HCW have the knowledge about how to take sample, when patient has suspicion of having tuberculosis. And about 50\% HCW have the general idea of what to do in case of blood spillage and disinfection.

\section{Post session}

There was significant increase in knowledge of how \& when to take sample i.e $58 \%$ \& $60 \%$ respectively which is almost more than double of pre session. Regarding storage of sample almost $80 \%$ gave correct answer. $60 \%$ of people now had knowledge of when to expect report. There was 3 times increase in knowledge of how to transport sample. $90 \%$ people had knowledge of processing of tuberculosis sample. The knowledge of blood spillage handling increase a little less i.e only $10 \%$.Overall training doubled the knowledge of $\mathrm{HCW}$

\begin{tabular}{|c|c|c|c|c|c|c|c|c|c|c|c|c|c|c|c|c|c|c|c|c|c|c|c|c|}
\hline \multirow{2}{*}{$\begin{array}{c}\text { S.N } \\
\mathbf{O}\end{array}$} & \multirow[t]{2}{*}{ NAME } & \multirow[t]{2}{*}{ POST } & \multicolumn{10}{|c|}{ PRE TEST SCORE } & \multirow{2}{*}{$\begin{array}{c}\text { TOTA } \\
\mathrm{L} \\
\end{array}$} & \multicolumn{10}{|c|}{ POST TEST SCORE } & \multirow{2}{*}{$\begin{array}{c}\text { TOTA } \\
\text { L }\end{array}$} \\
\hline & & & 1 & 2 & 3 & 4 & 5 & 6 & 7 & 8 & 9 & 10 & & 1 & 2 & 3 & 4 & 5 & 6 & 7 & 8 & 9 & 10 & \\
\hline 1 & $\begin{array}{l}\text { Sumit } \\
\text { kumar }\end{array}$ & Attendor & $\mathrm{Y}$ & $\mathrm{N}$ & $\mathrm{N}$ & $\mathrm{N}$ & $\mathrm{N}$ & $\mathrm{N}$ & $\mathrm{Y}$ & $\mathrm{N}$ & $\mathrm{N}$ & $\mathrm{N}$ & 2 & $\mathrm{Y}$ & $\mathrm{Y}$ & $\mathrm{N}$ & $\mathrm{Y}$ & $\mathrm{N}$ & $\mathrm{N}$ & $\mathrm{Y}$ & $\mathrm{N}$ & $\mathrm{N}$ & $\mathrm{N}$ & 4 \\
\hline 2 & $\begin{array}{l}\text { Deepak } \\
\text { Tiwari }\end{array}$ & $\begin{array}{l}\text { OPDAtte } \\
\text { ndor }\end{array}$ & $\mathrm{Y}$ & $\mathrm{N}$ & $\mathrm{N}$ & $\mathrm{N}$ & $\mathrm{N}$ & $\mathrm{N}$ & $\mathrm{N}$ & $\mathrm{N}$ & $\mathrm{N}$ & $\mathrm{N}$ & 1 & $\mathrm{Y}$ & $\mathrm{Y}$ & $\mathrm{N}$ & $\mathrm{N}$ & $\mathrm{N}$ & $\mathrm{N}$ & $\mathrm{N}$ & $\mathrm{N}$ & $\mathrm{N}$ & $\mathrm{N}$ & 2 \\
\hline 3 & $\begin{array}{l}\text { Dheeraj } \\
\text { Tiwari }\end{array}$ & $\begin{array}{c}\text { OPD } \\
\text { Attendor }\end{array}$ & $\mathrm{Y}$ & $\mathrm{N}$ & $\mathrm{N}$ & $\mathrm{N}$ & $\mathrm{N}$ & $\mathrm{N}$ & $\mathrm{N}$ & $\mathrm{N}$ & $\mathrm{N}$ & $\mathrm{N}$ & 1 & $\mathrm{Y}$ & $\mathrm{N}$ & $\mathrm{N}$ & $\mathrm{N}$ & $\mathrm{N}$ & $\mathrm{N}$ & $\mathrm{N}$ & $\mathrm{N}$ & $\mathrm{N}$ & $\mathrm{N}$ & 1 \\
\hline 4 & $\begin{array}{c}\text { Arun } \\
\text { Kumar }\end{array}$ & Attendor & $\mathrm{Y}$ & $\mathrm{N}$ & $\mathrm{N}$ & $\mathrm{N}$ & $\mathrm{N}$ & $\mathrm{N}$ & $\mathrm{Y}$ & $\mathrm{N}$ & $\mathrm{N}$ & $\mathrm{N}$ & 2 & $\mathrm{Y}$ & $\mathrm{N}$ & $\mathrm{N}$ & $\mathrm{Y}$ & $\mathrm{N}$ & $\mathrm{N}$ & $\mathrm{Y}$ & $\mathrm{Y}$ & $\mathrm{N}$ & $\mathrm{N}$ & 4 \\
\hline 5 & $\begin{array}{l}\text { Sheetal } \\
\text { Raikwar }\end{array}$ & $\begin{array}{c}\text { lab } \\
\text { Attendor }\end{array}$ & $\mathrm{Y}$ & $\mathrm{Y}$ & $\mathrm{N}$ & $\mathrm{N}$ & $\mathrm{Y}$ & $\mathrm{Y}$ & $\mathrm{Y}$ & $\mathrm{N}$ & $\mathrm{N}$ & $\mathrm{N}$ & 5 & $\mathrm{Y}$ & $\mathrm{N}$ & $\mathrm{N}$ & $\mathrm{N}$ & $\mathrm{Y}$ & $\mathrm{Y}$ & $\mathrm{Y}$ & $\mathrm{Y}$ & $\mathrm{N}$ & $\mathrm{N}$ & 5 \\
\hline 6 & $\begin{array}{l}\text { Bholu } \\
\text { Kumar }\end{array}$ & $\begin{array}{c}\text { Lab } \\
\text { Technicia } \\
n\end{array}$ & $\mathrm{Y}$ & $\mathrm{Y}$ & $\mathrm{N}$ & $\mathrm{N}$ & $\mathrm{Y}$ & $\mathrm{Y}$ & $\mathrm{Y}$ & $\mathrm{Y}$ & $\mathrm{N}$ & $\mathrm{N}$ & 6 & $\mathrm{Y}$ & $\mathrm{Y}$ & $\mathrm{N}$ & $\mathrm{N}$ & $\mathrm{Y}$ & $\mathrm{Y}$ & $\mathrm{Y}$ & $\mathrm{Y}$ & $\mathrm{N}$ & $\mathrm{N}$ & 6 \\
\hline 7 & $\begin{array}{l}\text { Devaki } \\
\text { Manjhi }\end{array}$ & $\begin{array}{c}\text { Lab } \\
\text { Attendor }\end{array}$ & $\mathrm{Y}$ & $\mathrm{Y}$ & $\mathrm{N}$ & $\mathrm{N}$ & $\mathrm{Y}$ & $\mathrm{Y}$ & $\mathrm{Y}$ & $\mathrm{N}$ & $\mathrm{N}$ & $\mathrm{N}$ & 5 & $\mathrm{Y}$ & $\mathrm{Y}$ & $\mathrm{N}$ & $\mathrm{N}$ & $\mathrm{Y}$ & $\mathrm{Y}$ & $\mathrm{Y}$ & $\mathrm{Y}$ & $\mathrm{N}$ & $\mathrm{N}$ & 6 \\
\hline 8 & $\begin{array}{l}\text { Chandra } \\
\text { Bhargava }\end{array}$ & Matron & $\mathrm{Y}$ & $\mathrm{N}$ & $\mathrm{N}$ & $\mathrm{Y}$ & $\mathrm{N}$ & $\mathrm{N}$ & $\mathrm{N}$ & $\mathrm{N}$ & $\mathrm{N}$ & $\mathrm{N}$ & 2 & $\mathrm{Y}$ & $\mathrm{Y}$ & $\mathrm{N}$ & $\mathrm{Y}$ & $\mathrm{Y}$ & $\mathrm{Y}$ & $\mathrm{Y}$ & $\mathrm{Y}$ & $\mathrm{N}$ & $\mathrm{Y}$ & 8 \\
\hline 9 & $\begin{array}{c}\text { Harimohan } \\
\text { Sharma }\end{array}$ & $\begin{array}{c}\text { Staff } \\
\text { Nurse }\end{array}$ & $\mathrm{Y}$ & $\mathrm{N}$ & $\mathrm{N}$ & $\mathrm{N}$ & $\mathrm{Y}$ & $\mathrm{N}$ & $\mathrm{Y}$ & $\mathrm{Y}$ & $\mathrm{N}$ & $\mathrm{N}$ & 4 & $\mathrm{Y}$ & $\mathrm{Y}$ & $\mathrm{N}$ & $\mathrm{Y}$ & $\mathrm{Y}$ & $\mathrm{Y}$ & $\mathrm{Y}$ & $\mathrm{Y}$ & $\mathrm{N}$ & $\mathrm{Y}$ & 8 \\
\hline 10 & $\begin{array}{c}\text { Priyanka } \\
\text { Shakya }\end{array}$ & $\begin{array}{c}\text { Staff } \\
\text { Nurse }\end{array}$ & $\mathrm{Y}$ & $\mathrm{Y}$ & $\mathrm{N}$ & $\mathrm{N}$ & $\mathrm{Y}$ & $\mathrm{Y}$ & $\mathrm{N}$ & $\mathrm{N}$ & $\mathrm{N}$ & $\mathrm{N}$ & 4 & $\bar{Y}$ & $\mathrm{Y}$ & $\mathrm{Y}$ & $\mathrm{Y}$ & $\mathrm{Y}$ & $\mathrm{Y}$ & $\mathrm{Y}$ & $\bar{Y}$ & $\mathrm{Y}$ & $\mathrm{Y}$ & 10 \\
\hline 11 & $\begin{array}{l}\text { Manisha } \\
\text { Pante }\end{array}$ & $\begin{array}{c}\text { Staff } \\
\text { Nurse }\end{array}$ & $\mathrm{Y}$ & $\mathrm{N}$ & $\mathrm{Y}$ & $\mathrm{N}$ & $\mathrm{N}$ & $\mathrm{N}$ & $\mathrm{Y}$ & $\mathrm{Y}$ & $\mathrm{N}$ & $\mathrm{N}$ & 4 & $\mathrm{~N}$ & $\mathrm{Y}$ & $\mathrm{Y}$ & $\mathrm{Y}$ & $\mathrm{Y}$ & $\mathrm{Y}$ & $\mathrm{Y}$ & $\mathrm{Y}$ & $\mathrm{Y}$ & $\mathrm{Y}$ & 9 \\
\hline 12 & Sonam Patil & $\begin{array}{c}\text { Sister } \\
\text { Incharge }\end{array}$ & $\mathrm{y}$ & $\mathrm{N}$ & $\mathrm{Y}$ & $\mathrm{N}$ & $\mathrm{Y}$ & $\mathrm{N}$ & $\mathrm{Y}$ & $\mathrm{N}$ & $\mathrm{N}$ & $\mathrm{N}$ & 4 & $\mathrm{Y}$ & $\mathrm{Y}$ & $\mathrm{Y}$ & $\mathrm{Y}$ & $\mathrm{Y}$ & $\mathrm{Y}$ & $\mathrm{Y}$ & $\mathrm{Y}$ & $\mathrm{Y}$ & $\mathrm{Y}$ & 10 \\
\hline 13 & Anil Baghel & $\begin{array}{c}\text { Lab } \\
\text { Technicia } \\
n\end{array}$ & $\mathrm{~N}$ & $\mathrm{~N}$ & $\mathrm{~N}$ & $\mathrm{~N}$ & $\mathrm{~N}$ & $\mathrm{~N}$ & $\mathrm{~N}$ & $\mathrm{~N}$ & $\mathrm{~N}$ & $\mathrm{~N}$ & & $\mathrm{Y}$ & $\mathrm{Y}$ & $\mathrm{N}$ & $\mathrm{Y}$ & $\mathrm{Y}$ & $\bar{Y}$ & $\mathrm{Y}$ & $\mathrm{N}$ & $\mathrm{Y}$ & $\mathrm{N}$ & 7 \\
\hline 14 & $\begin{array}{c}\text { Silvia } \\
\text { MLeonard }\end{array}$ & $\begin{array}{c}\text { Staff } \\
\text { Nurse }\end{array}$ & $\mathrm{N}$ & $\mathrm{N}$ & $\mathrm{N}$ & $\mathrm{N}$ & $\mathrm{N}$ & $\mathrm{N}$ & $\mathrm{N}$ & $\mathrm{N}$ & $\mathrm{N}$ & $\mathrm{N}$ & & $\mathrm{Y}$ & $\mathrm{Y}$ & $\mathrm{N}$ & $\mathrm{Y}$ & $\mathrm{Y}$ & $\mathrm{N}$ & $\mathrm{Y}$ & $\mathrm{Y}$ & $\mathrm{Y}$ & $\mathrm{Y}$ & 8 \\
\hline 15 & $\begin{array}{c}\text { Anil } \\
\text { Sharma }\end{array}$ & $\begin{array}{c}\text { ECG } \\
\text { Technicia } \\
n\end{array}$ & $\mathrm{~N}$ & $\mathrm{~N}$ & $\mathrm{~N}$ & $\mathrm{~N}$ & $\mathrm{~N}$ & $\mathrm{~N}$ & $\mathrm{~N}$ & $\mathrm{~N}$ & $\mathrm{~N}$ & $\mathrm{~N}$ & & $\mathrm{~N}$ & $\mathrm{Y}$ & $\mathrm{N}$ & $\mathrm{N}$ & $\mathrm{N}$ & $\mathrm{N}$ & $\mathrm{Y}$ & $\mathrm{Y}$ & $\mathrm{N}$ & $\mathrm{N}$ & 3 \\
\hline & & & $\begin{array}{l}1 \\
2\end{array}$ & 4 & 1 & 1 & 6 & 4 & 7 & 3 & 0 & 0 & & 13 & 12 & 3 & 9 & 10 & 9 & $\begin{array}{l}1 \\
3\end{array}$ & $\begin{array}{l}1 \\
1\end{array}$ & 5 & 6 & \\
\hline
\end{tabular}

\begin{tabular}{|l|l|l|l|l|l|}
\hline S.NO & MODE OF SAMPLE & \multicolumn{2}{l|}{ PRE TEST } & \multicolumn{2}{l|}{ POST TEST } \\
\hline 1 & How to collect sample & 12 & 26.6 & 26 & 57.8 \\
\hline 2 & When to collect sample & 1 & 6.7 & 9 & 60 \\
\hline 3 & Storage of sample & 4 & 26.7 & 12 & 80 \\
\hline 4 & Transportation of sample & 4 & 13.3 & 14 & 46.7 \\
\hline 5 & Processing time of sample & 4 & 26.7 & 9 & 60 \\
\hline 6 & Collection of sample in TB patients & 7 & 46.7 & 13 & 86.7 \\
\hline 7 & Handling of blood sample. & 6 & 50 & 10 & 66.7 \\
\hline
\end{tabular}




\section{Discussion}

Specimen collection plays an important role in getting timely and accurate results of investigation required for diagnosis. If there is any error in delivery of the sample from patients to laboratory, which might be due to improper collection, untimely collection, poor transportation, it will lead to error in diagnosis ${ }^{(1)}$ and most of these mistakes are human and mostly at pre-analytical level which are avoidable. Among them HCW play an important role who provide first hand bedside care and their action have a direct role in positive patient outcome as it affects sample quality which is processed in laboratory .

It is therefore important to develop systems and routine to enhance knowledge, attitude and practice among health care workers regarding proper sample collection, storage and transportation $^{(2)}$

\section{Dimorphic details}

\begin{tabular}{|l|c|}
\hline Characteristics & N (\%) \\
\hline Gender distribution & $53.3 \%$ \\
\hline Male & $46.2 \%$ \\
\hline Female & $40 \%$ \\
\hline Distribution of participants \\
\hline Nurses & $20 \%$ \\
\hline Technicians & $40 \%$ \\
\hline Attendants \\
\hline Age groups (in years) \\
\hline $21-30$ & $60 \%$ \\
\hline $31-40$ & $30 \%$ \\
\hline $41-50$ & $10 \%$ \\
\hline Educational qualification \\
\hline Bsc /diploma & $30 \%$ \\
\hline MLT & $60 \%$ \\
\hline Level of experience \\
\hline <than 5 years & $70 \%$ \\
\hline 5-10 years & $15 \%$ \\
\hline 10-15 years & $15 \%$ \\
\hline
\end{tabular}

In our study $8(53.3 \%)$ were male and $7(46.2 \%)$ were female. while study done by Chandak, poonam et al ${ }^{(3)}$ and mythriH ${ }^{1}$,Arun $\mathrm{A}^{2}$ and K.R. Kashinath $^{3(4)}$ Showed a higher percentage of female as compared to males.

In this study nurses were (40\%), technicians were (20\%) and attendant were (40\%). while study done by mythri $\mathrm{H}^{1}$, Arun $\mathrm{A}^{2}$ and K.R. Kashinath ${ }^{3(4)}$ Showed that nurses were( $53.9 \%)$,technicians were $(11.7 \%)$ and attendant were $(34.4 \%)$.
Majority of participants $(60 \%)$ were in the age group of $20-30$ years, $(30 \%)$ were in the age group of 31-40 years and (10\%) were in the age group of 41-50. Similar study done by mythri $\mathrm{H}^{1}$, Arun $\mathrm{A}^{2}$ andK.R.Kashinath ${ }^{3 .(4)}$

Majority of participants (60\%) had done MLT and (30\%) had done BSc/Diploma. Similar study done by Chandak, poonam et $\mathrm{al}^{(3)}$ where diploma holders were $89.9 \%$ and MLT were $10.1 \%$ While unlike our results study done bymythriH ${ }^{1}$,Arun $\mathrm{A}^{2}$ and K.R.Kashinath ${ }^{3(4)}$ where MLT participants were $(11.7 \%)$ and diploma/BSc were $(53.9 \%)$. None of the participants were post graduate.

In this study $70 \%$ were less than 5 years of experience, $15 \%$ were between 5-10 years, and $15 \%$ were between 10-15 years. Similar study done by mythri $\mathrm{H}^{1}$, Arun $\mathrm{A}^{2}$ and K.R. Kashinath $^{3(4)}$. While study done by Chandak, poonam et $\mathrm{al}^{(3)} 35.9 \%$ had upto 5 years experience, $49.4 \%$ 6-10years experience $10.1 \%$ had 11-15 years experience.

In our study the knowledge regarding how \&when to collect the sample, storage of sample, when to expect report, how to transport sample and processing of sample was increased by $70 \%$ .Similar study done by Chandak, poonam et al ${ }^{(3)}$ while study done by B. Sandeep et. $\mathrm{al}^{(5)}$ showed the increase of knowledge only $32 \%$.

In our study the knowledge regarding blood spillage handling increased only $10 \%$ while study done by B. Sandeep et al ${ }^{(5)}$ showed the increase of knowledge $30 \%$.

Over all training double the knowledge of HCW very few study on this.

\section{Conclusion}

Key to patient care is correct diagnosis and most of the error at pre analytical phase $(46 \%-68 \%$ of total error) i.e before the sample is analyzed. Analytical and post analytical errors are less frequent ${ }^{(2)}$. It is there for important to train $\mathrm{HCW}$, who form the root of health care system at regular intervals this enhance knowledge significantly. Further training session can be give to junior 
residents and clinicians who direct $\mathrm{HCW}$ and in turn affect sample quality.

\section{References}

1. Baron EJ, Peterson LR, Finegold SM. Selection, collection, and transport of specimens for microbial examination. Bailey and Scott's diagnostic microbiology. $9^{\text {th }}$ ed. St. Louis Mosby-year Book; 1994. P.53-64.

2. Khan F, Sami H, Rizvi M, Shah M S, Khan T, Ahmad M, Sultan A, Shukla I. Specimen collection: the art of laboratory science among the clinicians. Jpatient Saf Infect control 2017;5:35-9.

3. Chandak ,Poonam S.Loomba, B. Mishra, V.Dogra. Impact of training on knowledge and practice of Nurses Regarding Hospital Infection Control Practices in a tertiary care Hospital in Central, South India. NJIRM 2016; Vol 7(4):39-43.

4. Mythri $\mathrm{H}^{1}$, Arun $\mathrm{A}^{2}$, and K.R.Kashinath ${ }^{3}$ perception and practice regarding infection control measures among healthcare workers. JCPR 2015 Vol - 7(2); 109-114.

5. B. Sandeep et. al. Impact of training on knowledge and practice of Nurses Regarding Hospital Infection Control Practices in a tertiary care Hospital in Central India. IJAR. Vol -7(8); 233-34. Aug.2017.

6. Carraro P, Plebani M. Errors in a stat laboratory: Types and frequencies 10 years later, Clin Chem2007;53:1338-42.

7. Fang L,Fang SH. Chung YH, Chien ST. Collection factors related to the haemolysis of blood specimen. J Clin Nurs 2008;17:2343-51.

8. Bonini P, Plebani M, Cenotti F.Errors in laboratory medicine. Clin Chem 2002;48:691-8.
9. Plebani M,Errors in clinical laboratories or errors in laboratory medicine? Clin Chem Lab Med 2006;44:750-9.

10. Ojide CK, Onwuezobe IA, Asuquo EE, Obiagwu CS. Knowledge, attidude and practice of blood culture: A cross sectional study among medical doctors in a Nigerian tertiary hospital. Afr J Clin Exp microbio 2013;14:174-9. 